\title{
A Rare Case of Severe Dilated Cardiomyopathy in Early Infancy
}

\author{
Meike Schwendt ${ }^{1} \quad$ Johannes Kroll ${ }^{2}$ Thilo Fleck ${ }^{1}$ \\ ${ }^{1}$ Department of Congenital Heart Disease and Paediatric Cardiology, \\ Freiburg University Hospital, Freiburg, Baden-Württemberg, Germany \\ ${ }^{2}$ Department of Cardio-Thoracic Surgery at University Heart Centre \\ Freiburg, Freiburg University Hospital, Bad Krozingen, Medical \\ Centre, University of Freiburg, Faculty of Medicine, Freiburg, \\ Baden-Württemberg, Germany \\ Thorac Cardiovasc Surg Rep 2021;10:e12-e14.
}

Brigitte Stiller ${ }^{1}$

\author{
Address for correspondence Meike Schwendt, MD, Department of \\ Congenital Heart Disease and Paediatric Cardiology, Freiburg \\ University Hospital, Mathildenstr. 1, Freiburg 79106, \\ Baden-Württemberg, Germany \\ (e-mail: meike.schwendt@universitaets-herzzentrum.de).
}

\begin{abstract}
Keywords

- cardiomyopathy

- Alström syndrome

- Berlin Heart EXCOR

- heart transplantation

We report the case of a 3-month-old girl presenting with end-stage dilated cardiomyopathy and therapy-resistant cardiogenic shock. A left ventricular assist device (LVAD) Berlin Heart EXCOR was implanted, her organs recovered, and she was listed for heart transplantation. Two months later, while still on the LVAD, she was diagnosed with the rare genetic Alström syndrome. Weaning was no option, and she underwent successful heart transplantation at the age of 9 months. The follow-up 15 months later revealed an uneventful transplant course in a child with Alström syndrome.
\end{abstract}

\section{Introduction}

End-stage dilated cardiomyopathy (DCM) in otherwise apparently healthy young infants is associated with a poor prognosis. Acute cardiogenic shock forces intensivists and families to make an immediate, life-determining decision. The Berlin Heart EXCOR (Berlin, Germany) is the sole CE- and FDAmarked left ventricular assist device (LVAD) for longer term support until recovery or bridge to transplant in young infants.

Alström syndrome (ALMS) is a rare autosomal recessive disease caused by a mutation in the ALMS1 gene. It is characterized by multisystemic disorders, optionally including the risk of visual impairment, hearing loss, obesity, and diabetes.

\section{Case Presentation}

We report on a previously healthy female infant with nonconsanguine healthy parents who developed initial heartfailure symptoms in the form of feeding disorder, tachypnea, and failure to thrive at the age of 2 months. Four weeks later she was admitted to our intensive care unit (ICU), presenting in cardiogenic shock with multiple organ failure refractory to intravenous (IV) diuretics, milrinone, levosimendan, and catecholamines. The indication for LVAD support had therefore become urgently apparent.

With a body weight of only $3.4 \mathrm{~kg}$, the smallest available Berlin Heart EXCOR, LVAD with a stroke volume of $10 \mathrm{~mL}$ was implanted as a bridge to transplantation ( $\mathbf{- F i g . ~ 1 ) . ~ F o r ~}$ inflow, a $6 \mathrm{~mm}$ apex cannula and for outflow, a $6 \mathrm{~mm}$ endto-side aortic cannula (both Berlin Heart AG, Berlin, Germany) were implanted. Despite poor biventricular function at the time of VAD implantation, we decided to use an LVAD only. We supported the right ventricle with IV adrenalin, milrinone and inhaled NO, optimized $\mathrm{pH}$ and hemoglobin level, and took care not to "overpump" the circulation. So, we started with a pump rate of only 40 bpm and relied on continuous transesophageal echo to monitor RV function and careful unloading of the LV to reduce the afterload for the RV. During the subsequent 150 minutes, we raised the pump rate very slowly stepby-step from 40 up to $107 \mathrm{bpm}$ while still in the operating room (OR) (cardiopulmonary bypass 75 minutes, time in the OR 6 hours, 30 minute). received

August 4, 2020

accepted

September 9, 2020
DOI https://doi.org/ 10.1055/s-0040-1721038. ISSN 2194-7635.

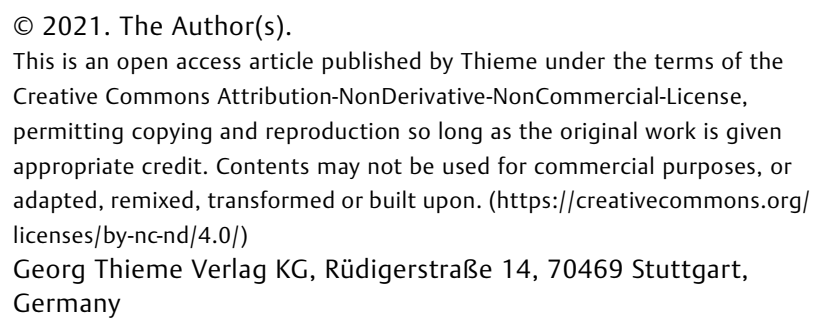


Myocardial biopsies revealed the full manifestation of DCM, but were otherwise inconspicuous regarding viralinflammatory or mitochondrial-related diseases.

Over the course, her situation stabilized on LVAD as all other organ functions recovered. After 120 hours on the ventilator and 23 days in the ICU, she was discharged to our normal pediatric cardiac ward. We put her on long-term anticoagulation with enoxaparin subcutaneously (target anti Xa $0.8-1.2 \mathrm{U} / \mathrm{L})$, acetylsalicylic acid $(4-5 \mathrm{mg} / \mathrm{kg} / \mathrm{d})$, clopidogrel $(0.1-0.2 \mathrm{mg} / \mathrm{kg} / \mathrm{d})$ and AT III substitution if below $80 \%$. No stroke or bleeding occurred over a 6-month support period; no pump exchange was necessary (-Fig. 1).

However, at the age of 6 months she developed photophobia and nystagmus, and began exhibiting minimally delayed developmental milestones while still on VAD.

The combination of heart failure and visual impairment in our infant implied an underlying syndromic and systemic disease. Once ALMS was genetically proven, we found no published follow-up reports on heart transplantation (HTx) in this rare patient population.

Due to ALMS' unpredictable individual course, we discussed the ethical aspects, prognosis, and potential complications with her parents. In accordance with the care keepers, our team decided to maintain our HTx course. Finally, after having been on LVAD for 173 days, the child underwent successful HTx in bicaval technique at the age of 9 months (-Fig. 2). Cold ischemic time was 180 minutes and total ischemic time was 224 minutes. With the donor weighing $13 \mathrm{~kg}$, the donor/ recipient ratio was 2.1/1.

Postoperative induction therapy basiliximab (Simulect) on days 1 and 4 and methylprednisolone (Urbason) for 72 hours were used for induction therapy after HTx. Additional immunosuppressive therapy includes cyclosporine (Sandimmun) and everolimus (Certican) in our department. The girl was discharged home 4 weeks after HTx in good clinical and cardiac condition on an immunosuppressive regime of prednisolone, everolimus, and cyclosporin.

Now, 1.5 years after HTx, she continues to present in good clinical and cardiac condition and there have been no clinical signs of rejection or infection in between. One-year routine angiography and myocardial biopsy revealed no signs of rejection. The right ventricle exhibited a mildly reduced diastolic function (right ventricular end-diastolic pressure $11 \mathrm{~mm} \mathrm{Hg}$ ) with moderately elevated pulmonary pressure (mean pulmonary arterial pressure $21 \mathrm{~mm} \mathrm{Hg}$ ). Left ventricular end-diastolic pressure was low $(8 \mathrm{~mm} \mathrm{Hg}$ ) and there were no signs of incipient transplant vasculopathy. Despite suffering severe vision loss, she is in fact developing almost like a normal child, walking free at the age of 24 months, revealing good verbal understanding, but speaking few words. She exhibits no further ALMS symptoms at the age of 28 months.

\section{Discussion and Conclusion}

ALMS is a rare (1:1 Mio) autosomal-recessive, potentially multisystemic disorder including early cone-rod dystrophy entailing progressive visual impairment, optional hearing
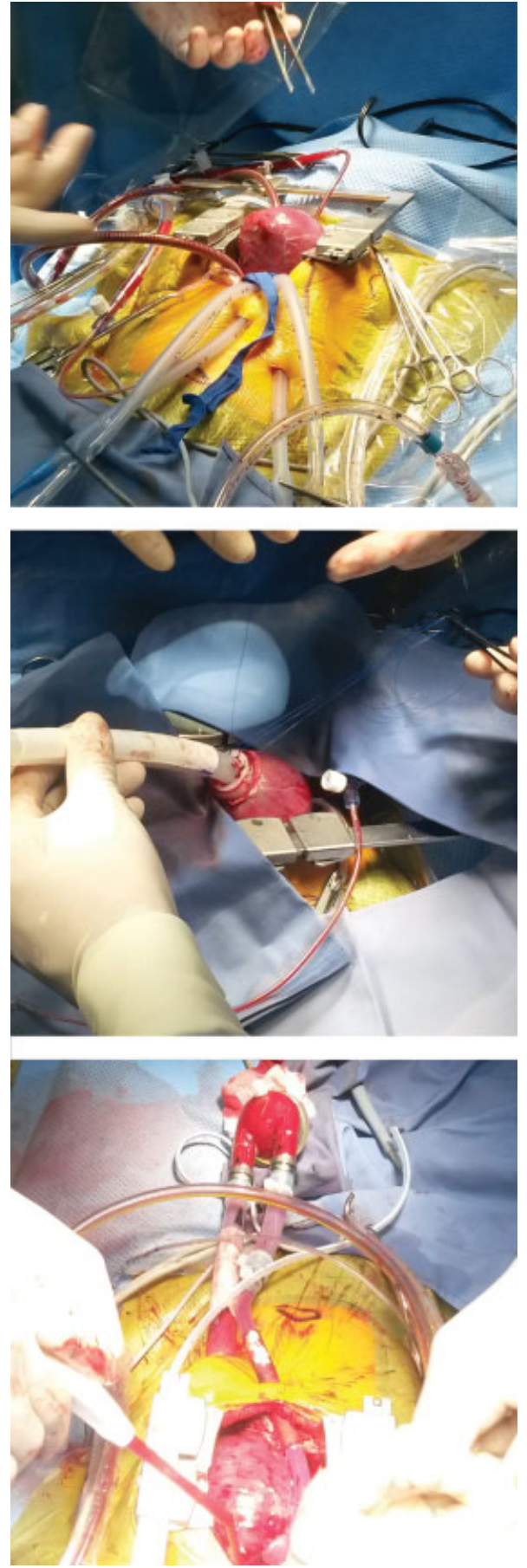

Fig. 1 Berlin Heart EXCOR left ventricular assist device (LVAD) implantation in the operating room. Top: open chest with enlarged heart due to dilated cardiomyopathy Center: implantation of Berlin Heart cannula into the apex of the left ventricle. Bottom: Berlin Heart LVAD implantation previous to chest closure.

loss, obesity, diabetes, and multiple organ fibrosis. ${ }^{1,2}$ This multisystemic disorder reveals widely varying symptoms and severity, and the current life expectancy rarely exceeds 50 years. $^{3}$

Located on chromosome 2p13, the ALMS I gene has an important function in endosomal and ciliary transport, cell cycle regulation and cell differentiation, with up to 239 mutations described in this gene. Currently, there is no 
e14 A Rare Case of Severe DCM in Early Infancy Schwendt et al.
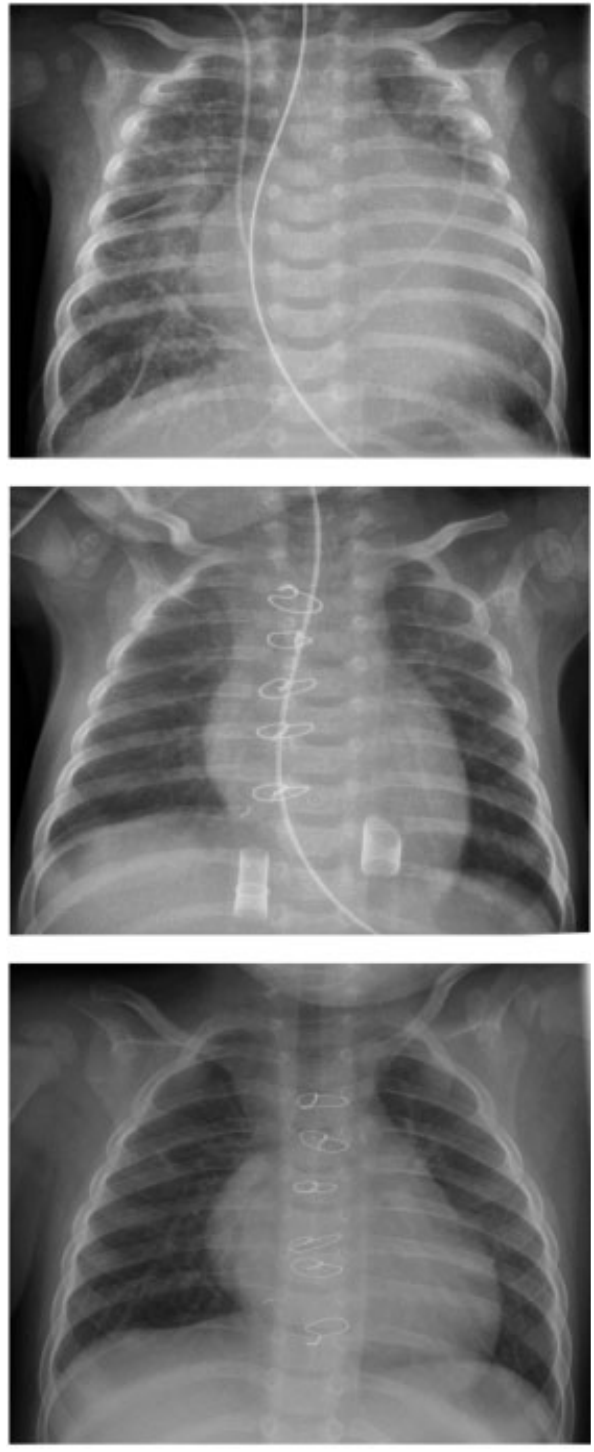

Fig. 2 Serial thoracic X-ray. Top: X-ray with severe cardiomegaly due to dilated cardiomyopathy. Center: unloaded heart with Berlin Heart cannulae in situ and a decline of cardiomegaly. Bottom: X-ray of chest after heart transplantation with a mildly oversized heart presenting almost normal size.

strong correlation between genotype and phenotype. ${ }^{4}$ The combination of mutated alleles is apparently important for this phenotype's appearance, accounting for this disorder's heterogeneity. ${ }^{2,4}$ ALMS' clinical diagnosis is difficult, as it is characterized by a constellation of progressive and highly variable symptoms that occur within the first months or later in life. Noticeable is the vast clinical variability of symptoms in the same family, reported even in monozygotic twins. ${ }^{5}$

Congestive heart failure due to DCM occurs in 60\%. It can be the first symptom and play a crucial role for these patients, and appear even before nystagmus. ${ }^{1}$ It can remarkably resolve in some cases and may recur in adolescence or adulthood. ${ }^{1,6}$ Mitogenic cardiomyopathy is a special form of DCM and often presents with ALMS. The ALMS I mutation impairs neonatal terminal differentiation of cardiomyocytes and leads to mitogenic cardiomyopathy. ${ }^{7,8}$

We report on this infant's encouraging course after cardiogenic shock due to DCM, followed by good motoric and psychomental development while on LVAD, exactly at the time the genetic diagnosis of ALMS was made. Ethical considerations and the outspoken parents; strong wishes led us to continue the HTx course. Since we had detected no previous reports on transplantation courses, immune suppression, or the post-transplant development of ALMS children, we believe this case deserves to be reported.

\section{Conflict of Interest}

None.

\section{References}

1 Marshall JD, Beck S, Maffei P, Naggert JK. Alström syndrome. Eur J Hum Genet 2007;15(12):1193-1202

2 Álvarez-Satta M, Castro-Sánchez S, Valverde D. Alström syndrome: current perspectives. Appl Clin Genet 2015;8:171-179

3 Marshall JD, Maffei P, Collin GB, Naggert JK. Alström syndrome: genetics and clinical overview. Curr Genomics 2011;12(03): 225-235

4 Marshall JD, Muller J, Collin GB, et al. Alström syndrome: mutation spectrum of ALMS1. Hum Mutat 2015;36(07):660-668

5 Hollander SA, Alsaleh N, Ruzhnikov M, et al. Variable clinical course of identical twin neonates with Alström syndrome presenting coincidentally with dilated cardiomyopathy. Am J Med Genet 2017;173(06):1687-1689

6 Brofferio A, Sachdev V, Hannoush H, et al. Characteristics of cardiomyopathy in Alström syndrome: prospective single-center data on 38 patients. Mol Genet Metab 2017;121(04):336-343

7 Hearn T. ALMS1 and Alström syndrome: a recessive form of metabolic, neurosensory and cardiac deficits. J Mol Med (Berl) 2019;97(01):1-17

8 Shenje LT, Andersen P, Halushka MK, et al. Mutations in Alström protein impair terminal differentiation of cardiomyocytes. Nat Commun 2014;5:3416 